\title{
¿Cómo puede verse afectado el personal de salud ante un error en la atención al sujeto de cuidado, que conlleve a la generación de un evento adverso centinela?
}

\begin{abstract}
How can healthcare personnel be affected by an error made when caring for a patient that results in the generation of a sentinel event? Como o pessoal de saúde pode ser afetado por um erro na atenção ao paciente, o que leva à geração de um evento sentinela?
\end{abstract}

Existen múltiples factores que día a día, durante la atención médica, pueden acarrear fallas en la seguridad del paciente, las cuales pueden causar algún tipo de daño en la salud de este. De acuerdo con la revista científica British Medical Journal, tras un estudio realizado por expertos, indica que los errores médicos son la tercera causa de muerte en EE. UU. después de las enfermedades cardiovasculares y el cáncer, con unos 250,000 fallecimientos anuales (1). Situación preocupante que ha encendido las alarmas desde el año 1999. En respuesta a ello, la OMS ha venido emitiendo una serie de políticas y lineamientos en seguridad del paciente que pretenden minimizar los principales riesgos para que no sucedan este tipo de eventos.

Por otro lado, detrás de cada situación insegura hay un profesional de la salud vinculado al evento, quien, a su vez, se ve afectado por lo general en lo emocional, laboral y en la parte legal. Esta figura es denominada SEGUNDA VÍCTIMA. Ahora bien, ¿Cómo se acompaña a estas personas en estas situaciones? La literatura ya enuncia algunas acciones, como apoyo y seguimiento psicológico, cambio de puesto de trabajo; sin embargo, esencialmente se requiere de un procedimiento institucional y de un equipo interdisciplinario que aborde la problemática desde antes, durante y después del evento para incidir con ello en las consecuencias que estas puedan traer. Existen elementos esenciales que no pueden faltar dentro del proceso, las cuales permiten facilitar el abordaje de la situación y el acompañamiento del colaborador, aspectos que se presentarán ampliamente en uno de los talleres a ofrecer en el XVI Congreso Internacional de la Facultad de Ciencias de la Salud "Manejo integral del paciente hospitalario" de la Universidad Autónoma de Bucaramanga UNAB - Colombia.

En Colombia, en el año 2008, el Ministerio de Salud y Protección Social, siendo consciente de la problemática antes mencionada y adhiriéndose a las directrices de la Organización Mundial de la Salud OMS, emite una política de seguridad del paciente e integra como requisito de habilitación la obligatoriedad de desarrollar el programa de seguridad del paciente indicando los elementos mínimos que este debe contener para que, desde un enfoque integral, se incluya en forma transversal dichos lineamientos en todo el proceso de atención. Entre los elementos 
que indica se encuentra la gestión del evento adverso, que al mirarlo en su globalidad, además de detectar, reportar y analizar los hallazgos, debe tener claro qué acciones se deben seguir cuando se presenten, especialmente, los eventos denominados centinelas, los cuales son definidos como "evento adverso grave que ocasiona prolongación de la estancia hospitalaria, algún tipo de incapacidad transitoria o permanente o la muerte y que requiere de intervención organizacional inmediata para evitar su recurrencia" (2).

De estos eventos, por la magnitud de las consecuencias que le acarrea al paciente, hay que tener claridad sobre la respuesta inmediata que se debe realizar, en donde esté contemplado la intervención a gestionar con el paciente y su familia y, por consiguiente, la de la segunda víctima, es decir, la del profesional de la salud que esté implicado en el evento.

Unido a ello, además de tenerlo incluido el sistema de salud, también lo tiene contemplado legalmente el Sistema de seguridad y salud del trabajo dentro del programa de higiene y seguridad, el cual debe, en conjunto con el programa de seguridad del paciente, establecer las acciones a realizar ante estas eventualidades, en donde se tengan estipuladas unas acciones de choque y otras de continuidad y seguimientos en el después.

Todo debe partir de la esencia del programa de seguridad del paciente, el cual debe fomentar una cultura no punitiva y justa; con una perspectiva global de mejora de los procesos asistenciales, y evitar culpar a los profesionales de la salud. Es decir, se debe favorecer el derecho que tiene el trabajador a un tratamiento respetuoso, comprensivo y compasivo, unido a un apoyo continuo.

Es por ello que el plan de acción frente a las segundas víctimas debe ser flexible, debe atender a las circunstancias del evento adverso, y debe ejecutarse escalonadamente, en donde, inicialmente, se debe dar manejo al servicio implicado, donde los colegas y el equipo de salud den el primer apoyo. Una segunda intervención es la brindada por parte del área de gestión de talento humano, quien da sustento psicológico y laboral. La tercera es a nivel organizacional, donde se da soporte jurídico. De esta manera, se ayuda integralmente a ese funcionario.

Es importante tener presente que la forma como se den estas intervenciones se va a ver afectada positiva o negativamente la organización, por lo tanto, no hay que improvisar.

Cualquier profesional de la salud puede estar vinculado en un evento adverso que le genere consecuencias según la complejidad del desenlace que la falla contrajo. Puede ir desde un llamado de atención por parte del comité de ética de la profesión, retiro temporal o permanente de la tarjeta profesional, hasta el cumplimiento de penas a nivel carcelario y económicos. Sin embargo, los daños morales y psicológicos a veces son los más difíciles de superar. Por ello es importante estar preparados para este tipo de eventos y, de esta manera, limitar las consecuencias negativas, lesivas y favorecer la gestión de lecciones aprendidas hacia el mejoramiento. Es necesario no esperar a que le suceda un hecho, es pertinente documentarse, asesórese $\mathrm{y}$, en especial, fortalecer la cultura de seguridad del paciente hacia una forma proactiva, en donde se gestionen los riesgos con antelación y se minimicen las fallas.

Claudia Blanco Vargas Enfermera, Especialista en Docencia Universitaria, Auditoria en Salud, Humanización de la Salud y de la Intervención Social. Universidad de Ciencias Aplicadas y Ambientales (UDCA), Universidad Autónoma de Manizales

\section{Referencias}

1. Martin M. and Michael D. Medical error is third biggest cause of death in the US, Revista British Medical Journal (BMJ). [Internet]. 2016 [citado 03 de febrero de 2019]. Recuperado a partir de: http://www.bmj.com/company/wp-content/uploads/2016/05/medical-errors.pdf

2. Ministerio de Salud y Protección Social. Herramientas para promover la estrategia de la Seguridad del Paciente en el Sistema Obligatorio de Garantía de Calidad de la Atención en Salud. [Internet]. 2007 [citado 03 de febrero de 2019]. Recuperado a partir de: https://www.minsalud.gov.co/sites/rid/1/Herramientas\%20para \%20la\%20Seguridad $\% 20$ del $\% 20$ Paciente.pdf 